\title{
Diagnostic Impact of Bone-Subtraction CT Angiography for Patients with Acute Subarachnoid Hemorrhage
}

\author{
P. Aulbach, D. Mucha, K. Engellandt, K. Hädrich, (DM. Kuhn, and R. von Kummer
}

\begin{abstract}
BACKGROUND AND PURPOSE: Detection and evaluation of ruptured aneurysms is critical for choosing an appropriate endovascular or neurosurgical intervention. Our aim was to assess whether bone-subtraction CTA is capable of guiding treatment for cerebral aneurysms in patients with acute SAH and could replace DSA.
\end{abstract}

MATERIALS AND METHODS: We prospectively studied 116 consecutive patients with SAH with 16-detector row bone-subtraction CTA and DSA before intracranial aneurysm treatment. Two independent neuroradiologists reviewed the bone-subtraction CTA blinded to DSA (reference standard). We determined the accuracy of bone-subtraction CTA for aneurysm detection and the measurement of aneurysm dimensions and compared the radiation doses of the 2 imaging modalities.

RESULTS: Seventy-one patients (61\%) had 74 aneurysms on DSA. Bone-subtraction CTA detected 73 of these aneurysms, but it detected 1 additional aneurysm. On a per-aneurysm basis, sensitivity, specificity, and positive and negative predictive values for bone-subtraction CTA were $99 \%, 98 \%$, and $99 \%$ and $98 \%$, respectively. For aneurysms of $\leq 3 \mathrm{~mm}$, sensitivity was $94 \%$ ( $95 \% \mathrm{Cl}, 73 \%-99 \%)$. Bone-subtraction CTA slightly overestimated neck and dome diameters by $<0.2 \mathrm{~mm}$ and overestimated the dome-to-neck ratios by $2 \%$ on average. Dose-length product was $565 \pm 201 \mathrm{mGy} \times \mathrm{cm}$ for bone-subtraction CTA and $1609 \pm 1300 \mathrm{mGy} \times \mathrm{cm}$ for DSA.

CONCLUSIONS: Bone-subtraction CTA is as accurate as DSA in detecting cerebral aneurysms after SAH, provides similar information about aneurysm configuration and measures, and reduces the average effective radiation dose for vascular diagnostics by $65 \%$. Diagnostic equivalence in association with dose reduction suggests replacing DSA with bone-subtraction CTA in the diagnostic work-up of spontaneous SAH.

ABBREVIATIONS: BSCTA = bone-subtraction CTA; D/N = dome-to-neck; NECT = nonenhanced CT

$\mathbf{P}$ rompt detection and evaluation of ruptured intracranial aneurysms is critical for choosing an appropriate endovascular or neurosurgical intervention. ${ }^{1}$ Invasive digital subtraction angiography carries an overall risk of neurologic complications, resulting in permanent deficits in $0.5 \%{ }^{2,3}$ Providing false-negative results in $5 \%-10 \%$ of patients, ${ }^{4}$ it also may increase the risk of rebleeding. ${ }^{5,6}$

Multidetector CT angiography with high spatial resolution and bone-subtraction CTA (BSCTA) approaches the diagnostic accuracy of DSA in the detection of intracranial aneurysms. ${ }^{7-12}$

Received January 29, 2015; accepted after revision June 22.

From the Department of Neuroradiology (P.A., K.E., K.H., R.v.K.), University Hospital Carl Gustav Carus, Technische Universität, Dresden, Germany; Institute for Medical Informatics and Biometry at the Medical Faculty (M.K.), Technische Universität, Dresden, Germany; and Department of Neuroradiology (D.M.), Heinrich Braun Hospital, Zwickau, Germany.

Please address correspondence to Rüdiger von Kummer, MD, Department of Neuroradiology, University Hospital Carl Gustav Carus, Technische Universität, Fetscherstr 74, 01307 Dresden, Germany; e-mail: ruediger.vonkummer@uniklinikumdresden.de

-- Indicates open access to non-subscribers at www.ajnr.org

http://dx.doi.org/10.3174/ajnr.A4497
Thus, BSCTA can be considered an alternative to DSA in treatment planning. ${ }^{13,14}$ Some authors already recommend BSCTA as the primary imaging in acute SAH. ${ }^{7,15,16}$ However, it still seems unclear whether BSCTA can provide sufficient information for therapy decisions, making diagnostic DSA redundant. ${ }^{17,18}$

We therefore tested the hypothesis that BSCTA is as accurate as DSA for the identification and characterization of cerebral aneurysms in patients with SAH, even for small aneurysms and for those at the level of the skull base. We additionally studied the reliability of BSCTA and radiation-exposure reduction by avoiding diagnostic DSA.

\section{MATERIALS AND METHODS Participants}

After University Hospital Dresden review board approval (EK No. 73042008 ) and informed consent, from November 2007 to June 2011, neuroradiologists or neurosurgeons familiar with the protocol prospectively enrolled patients with acute SAH able to undergo CTA and DSA. We classified SAH severity with the Fisher score. Patients underwent nonenhanced CT (NECT) and BSCTA 
Table 1: Scan parameters for CT examinations

\begin{tabular}{lccc}
\hline & & \multicolumn{2}{c}{ BSCTA } \\
\cline { 3 - 4 } \multicolumn{1}{c}{ Scan Parameters } & \multicolumn{3}{c}{ Low-Dose } \\
\hline Tube voltage $(\mathrm{kV})$ & 120 & 100 & 100 \\
Tube current (effective mAs) & 320 & 70 & 140 \\
Rotation time (sec) & 1.0 & 0.5 & 0.5 \\
Section acquisition $(\mathrm{mm})$ & $16 \times 1.5$ & $16 \times 0.75$ & $16 \times 0.75$ \\
Table speed/rotation $(\mathrm{mm})$ & 13.2 & 13.8 & 13.8 \\
Recon. section thickness $(\mathrm{mm})$ & 8 & 1.0 & 1.0 \\
Reconstruction increment $(\mathrm{mm})$ & 8 & 0.5 & 0.5 \\
Automated exposure control & Off & Off & Off \\
Contrast material $(\mathrm{mL})^{\mathrm{a}}$ & $\mathrm{NA}$ & $\mathrm{NA}$ & 80 \\
Injection rate $(\mathrm{mL} / \mathrm{s})$ & $\mathrm{NA}$ & NA & $4-5$ \\
\hline
\end{tabular}

Note:- Recon. indicates reconstruction; NA, not applicable.

a Iopromide, Ultravist, 370 mg I/mL; Bayer HealthCare, Berlin, Germany.

followed by DSA with 3D reconstructions. We documented the time interval between CT and DSA. Patients with typical exclusion criteria for CTA or previous coiling or clipping were excluded. Because we aimed to assess the accuracy of BSCTA for the detection and description of cerebral aneurysms, we did not follow patients with perimesencephalic SAH further.

\section{CTA Imaging Technique}

Examinations were performed with the patient in the supine position from the $\mathrm{C} 1$ vertebral body to the vertex on a 16-detector row spiral CT (Somatom Sensation 16; Siemens, Malvern, Pennsylvania). We performed BSCTA after low-dose NECT (bone mask) and CTA, avoiding motion by head fixation and minimizing the delay between the 2 scans.

We did not use standard NECT for bone masking because standard NECT is acquired with a gantry tilt preventing direct $\mathrm{x}$-ray to the eye lenses, whereas CTA data are acquired with no gantry tilt. The BSCTA algorithm used requires similar, narrow, section acquisition ( $0.75 \mathrm{~mm}$ for high spatial resolution) and datasets with minimum motion artifacts $(0.5$ seconds for minimized motion artifacts during acquisition). Standard-dose NECT, however, requires a slower rotation time of 1.0 second (to collect a sufficient amount of x-ray quanta) and a wider collimation of 1.5 $\mathrm{mm}$. The CT scan parameters are listed in Table 1. The acquisition time was approximately 10 seconds. Bone-subtraction was performed automatically by using special prototype software on a workstation (syngo 2006G and syngo MultiTechnique Workplace, VE31D; Siemens).

\section{DSA and 3D-DSA}

We used a rotational biplane DSA unit (Allura Xper FD 20 biplane; Philips Healthcare, Best, the Netherlands) for panangiography (all cerebral arteries). Per acquisition, we administered 3-6 mL of nonionic contrast agent (iohexol, Accupaque, $300 \mathrm{mg} \mathrm{I} / \mathrm{mL}$; GE Healthcare, Milwaukee, Wisconsin). 3D reformatted images of rotational angiographic data were generated at the DSA workstation.

\section{Image Analysis}

Two neuroradiologists (D.M., K.E.), with 14 and 10 years of experience, reviewed the DSA images independently and blinded to BSCTA. Readers were informed of the patient's clinical symptoms and initial CT findings. Reading of BSCTA and DSA datasets was separated by $8-10$ weeks to prevent bias. To assess intraobserver reliability, reader 1 analyzed 15 randomly selected BSCTA imaging studies (12.9\%) twice, separated by an interval of 1 month.

An aneurysm was considered entirely characterized if all 3 orthogonal dimensions were obtained and the aneurysm neck and dome and arterial incorporations into the sac or neck were visualized and precisely measured. We categorized aneurysms as "berry-formed," "fusiform," and "branching" if they were located at an arterial bifurcation.

The readers generated maximum intensity projections, volume rendering technique reformations, and multiplanar reconstructions searching for aneurysms. If multiple aneurysms were detected, the most likely source of bleeding was estimated. Dome-to-neck $(\mathrm{D} / \mathrm{N})$ ratios were calculated for both modalities. The 2 readers evaluated images in consensus in case of discrepancies. We evaluated the effect of patient motion on image quality for DSA and BSCTA and rated the quality of BSCTA images on a 4-point scale as "excellent," "good," "moderate," or "poor."

\section{Radiation Dose}

We measured the dose-length product of both modalities and calculated the effective doses. Among the recorded values for DSA examinations, we only considered the diagnostic portion of the dose-length product for comparison. Exposure information was reported automatically, as required by the standard of the International Electrotechnical Commission (IEC 60601-2-43) for total fluoroscopy time in minutes, total number of exposures in numbers, accumulated fluoroscopy dose in milligrays, accumulated exposure dose in milligrays, total dose in milligrays, total number of frames in numbers, image-area dose product in milligrays, entrance dose and air kerma in milligrays, exposure start time, kilovolt (peak), distance source-to-image receptor distance, exposure time, $\mathrm{x}$-ray tube current, positioner primary angle, positioner secondary angle, and frame rate.

Examination and dose reporting for both BSCTA and DSA examinations are provided through Radiology Information DICOM 2-way interface, by using the DICOM Worklist Management and Technique Performed Procedure Step Standards.

\section{Statistical Methods}

We used MedCalc for Windows 12 (Version 12.3.0; MedCalc Software, Mariakerke, Belgium) for statistical analysis. We considered DSA, including 3D reconstructions, as the reference standard for aneurysm evaluation. We calculated the sensitivity, specificity, and accuracy of BSCTA on per-aneurysm and per-patient bases and used the Wilson procedure, without a correction for continuity, for the limits of the CI. We used the Cohen $\kappa$ to quantify inter- and intrareader agreement beyond chance in detecting aneurysms with BSCTA. A $P$ value $\leq .05$ was statistically significant. We compared the DSA and BSCTA differences for aneurysm dome and neck diameter and the $\mathrm{D} / \mathrm{N}$ ratio, applying the Bland-Altman method and a paired Student $t$ test, to analyze differences in radiation doses.

\section{RESULTS}

\section{Participants}

During 44 months, 269 consecutive patients presented to our department with SAH. We excluded 6 patients with prior clipping or 
coiling and 147 patients due to imaging protocol violations. Finally, 116 patients (50\% women) (mean age, $53.9 \pm 13.6$ years) were prospectively examined with BSCTA and DSA according to the study protocol.

\section{CT Findings}

All patients had SAH, with a Fisher grade 1 in 8 patients (6.9\%), 2 in 7 patients $(6.0 \%), 3$ in 42 patients $(36.2 \%)$, and 4 in 59 patients (50.9\%).

\section{DSA Reference}

The time between BSCTA and DSA varied from 20 minutes to 43 hours (median time, 7.0 hours). Of 116 patients, 71 patients $(61.2 \%)$ had 74 intracranial aneurysms on initial DSA. Table 2

Table 2: Location and sizes of cerebral aneurysms as detected by DSA

\begin{tabular}{lccc}
\hline & \multicolumn{3}{c}{ Size Categories } \\
\cline { 2 - 4 } Location & $\begin{array}{c}\text { Small } \\
(\leq 3.0 \mathrm{~mm})\end{array}$ & $\begin{array}{c}\text { Medium } \\
(3.1-5.0 \mathrm{~mm})\end{array}$ & $\begin{array}{c}\text { Large } \\
(>5 \mathrm{~mm})\end{array}$ \\
\hline ICA & 0 & 0 & 1 \\
Infraclinoid & 0 & 0 & 1 \\
Ophthalmic & 1 & 6 & 2 \\
PcomA & 1 & 0 & 0 \\
Carotid-T & & & \\
ACA & 0 & 1 & 0 \\
A1 & 5 & 16 & 7 \\
A1/A2 & $3^{\mathrm{a}}$ & 0 & 3 \\
A2 and A3 & & & 2 \\
MCA & $4^{\mathrm{b}}$ & 8 & 1 \\
Trifurcation & 0 & 1 & 3 \\
PCA & & 0 & 2 \\
BA & 1 & 3 & 1 \\
Oral & & 0 & 23 \\
VA & 0 & 35 & \\
PICA & 0 & 15 & \\
V4 & 15 &
\end{tabular}

Note:-PcomA indicates posterior communicating artery; Carotid-T, intracranial ICA bifurcation; ACA, anterior cerebral artery; A1, A2, A3, segments of the ACA; PCA, posterior cerebral artery; BA, basilar artery; VA, vertebral artery; PICA, posterior inferior cerebellar artery; V4, distal segment of VA.

a One aneurysm missed by bone-subtraction CTA

${ }^{b}$ One aneurysm missed by DSA but detected by bone-subtraction CTA.
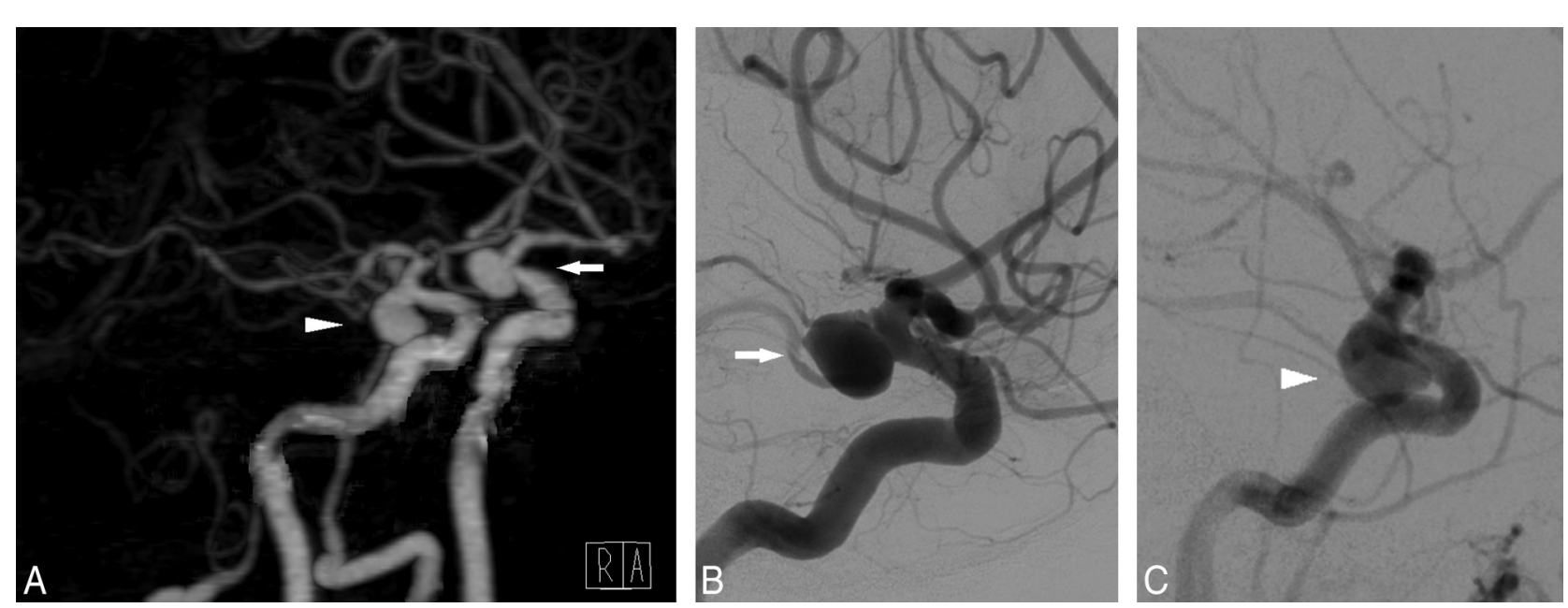

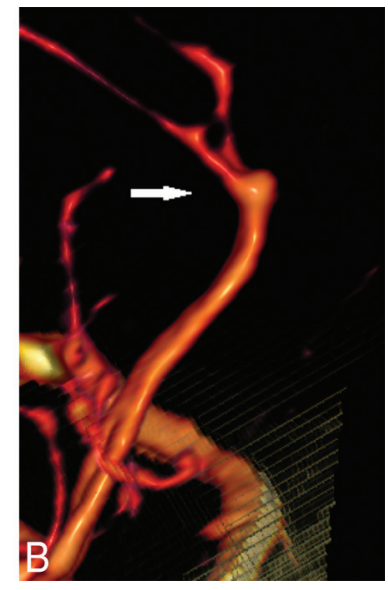

FIG 2. False-negative bone-subtraction CTA findings of an aneurysm of the right anterior cerebral artery in a 50-year-old woman. $A$, The right anterior oblique projection DSA shows a small broad-based aneurysm (arrow). B, On volume-rendering reconstruction, the aneurysm (arrow) appears fusiform. The white surface in the lower part of the image represents the bone-to-vessel boundary of the bone-removal processing.

FIG 1. A 78-year-old-female patient with symmetric infraclinoid aneurysms of the ICA. A, Volume-rendering of BSCTA displays both aneurysms (arrowhead: right aneurysm; arrow: left aneurysm). B and C, DSA confirms both aneurysms in size and configuration. 
MCA M2 segment aneurysm that was missed by DSA and finally confirmed by both readers in consensus (Fig 3). Both findings did not change clinical decisions.
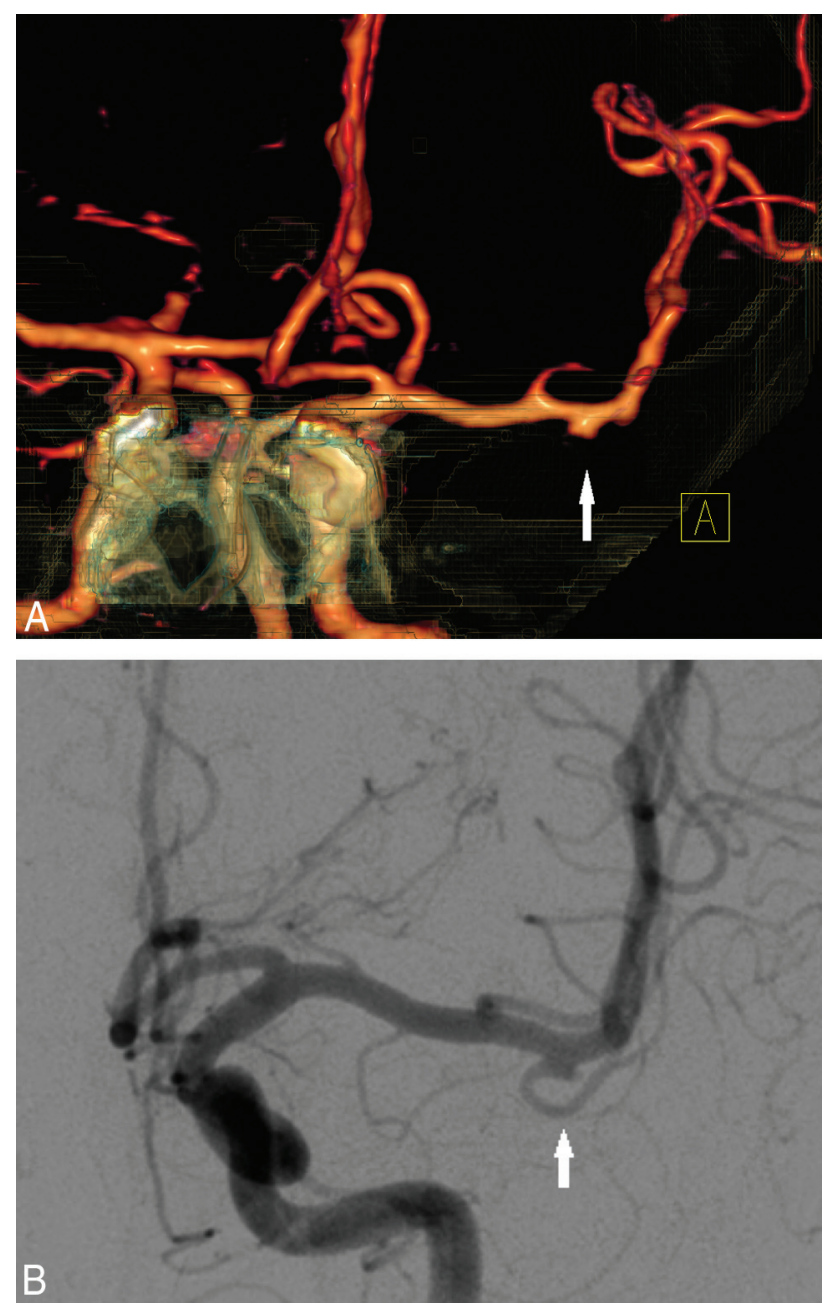

FIG 3. False-positive bone-subtraction CTA findings in an aneurysm $(2.5$ $\mathrm{mm}$ ) of the left middle cerebral artery distal to the trifurcation in a 50year-old man with 2 aneurysms. A, Volume-rendering of bone-subtraction CTA depicts the aneurysm (anteroposterior view) that was missed by DSA. B, 3D-DSA image of the initially missed M2 MCA trifurcation aneurysm (left anterior oblique view) that was confirmed in retrospect.
Sensitivity and specificity of BSCTA for intracranial aneurysms are presented in Tables 3 and 4.

Aneurysm dome diameters were slightly longer with a 0.17-mm bias ( $95 \% \mathrm{CI},-0.04$ to $0.39 \mathrm{~mm}$ ) measured on BSCTA compared with DSA (Fig 4A). The measurement differences are relatively constant over all aneurysm diameters. Aneurysm neck diameters of BSCTA did not differ from those on DSA (Fig 4B). Figure $4 \mathrm{C}$ shows that BSCTA aneurysm D/N ratios differ by -0.04 only ( $95 \% \mathrm{CI},-0.16$ to 0.08 ) from the DSA $\mathrm{D} / \mathrm{N}$ ratios. The average DSA $\mathrm{D} / \mathrm{N}$ ratio was $1.90 \pm 0.86$, and the average BSCTA D/N ratio was $1.86 \pm 0.84$. On average, BSCTA $\mathrm{D} / \mathrm{N}$ ratios were $2 \%$ smaller than DSA D/N ratios $(P=.4678)$. The highest agreement of methods was for the $\mathrm{D} / \mathrm{N}$ ratio of 1.5-2.0. Comparison of the distribution of $\mathrm{D} / \mathrm{N}$ ratios in DSA versus BSCTA is shown in Fig 4C.

\section{Image Quality and Reliability}

Overall bone-subtraction quality was high $(91.4 \%$ good or excellent). Ten of 116 datasets were rated moderate or poor. Fifteen of 74 aneurysms were near or surrounded by bone and were correctly detected and characterized with BSCTA. Motion artifacts impaired DSA in 28 patients $(23.9 \%)$ and BSCTA in 2 patients $(1.7 \%)$. The effect of the motion-impaired data on the diagnosis is shown in Table 5. The interobserver agreement for the identification of aneurysms was substantial $(\kappa=0.950$; 95\% CI, 0.894-1.000). Agreement per patient was also high with $(\kappa=0.965 ; 95 \%$ CI, 0.916-1.000). Both readers agreed substantially on aneurysm configuration $(\kappa=0.969 ; 95 \% \mathrm{CI}$, 0.941-0.996; $P<.001)$. Reader 1 agreed in all repeated cases with his initial reading.

\section{Radiation Dose}

The mean dose-length product was $564.7 \pm 201.4 \mathrm{mGy} \times \mathrm{cm}$ for BSCTA and $1608.9 \pm 1299.6 \mathrm{mGy} \times \mathrm{cm}$ for DSA, meaning a reduction of $65 \%$ ( $P<.001,2$-sample Student $t$ test $)$. The effective dose for BSCTA was between 0.8 and $3.6 \mathrm{mSv}$, with an average effective dose of $1.3 \pm 0.3 \mathrm{mSv}$. The average effective radiation dose for diagnostic DSA was $3.7 \pm 2.98 \mathrm{mSv}$, ranging from 0.37 to $17.3 \mathrm{mSv}$.

Table 3: Accuracy of BSCTA in detecting cerebral aneurysms

\begin{tabular}{cccccccccc}
\hline & $\begin{array}{c}\text { TP } \\
\text { (No.) }\end{array}$ & $\begin{array}{c}\text { TN } \\
\text { (No.) }\end{array}$ & $\begin{array}{c}\text { FP } \\
\text { (No.) }\end{array}$ & $\begin{array}{c}\text { FN } \\
\text { (No.) }\end{array}$ & $\begin{array}{c}\text { Sensitivity (\%) } \\
\text { (No.) }\end{array}$ & $\begin{array}{c}\text { Specificity (\%) } \\
\text { (No.) }\end{array}$ & $\begin{array}{c}\text { PPV (\%) } \\
\text { (No.) }\end{array}$ & $\begin{array}{c}\text { NPV (\%) } \\
\text { (No.) }\end{array}$ & $\begin{array}{c}\text { Accuracy (\%) } \\
\text { (No.) }\end{array}$ \\
\hline Per patient & 70 & 45 & 0 & 1 & $99(70 / 71)$ & $100(45 / 45)$ & $100(70 / 70)$ & $98(45 / 46)$ & $99(115 / 116)$ \\
$95 \%$ Cl & & & & & $92-100$ & $92-100$ & $95-100$ & $89-100$ & $95-100$ \\
Per aneurysm & 73 & 45 & 1 & 1 & $99(73 / 74)$ & $98(45 / 46)$ & $99(73 / 74)$ & $98(45 / 46)$ & $98(118 / 120)$ \\
95\% Cl & & & & & $93-100$ & $89-100$ & $93-100$ & $89-100$ & $94-100$ \\
\hline
\end{tabular}

Note:-TP indicates true-positive; TN, true-negative; FP, false-positive; FN, false-negative; PPV, positive predictive value; NPV, negative predictive value.

Table 4: Accuracy of BSCTA in detecting cerebral aneurysms of different sizes

\begin{tabular}{|c|c|c|c|c|c|c|c|c|c|}
\hline Diameter & $\begin{array}{l}\text { TP } \\
\text { (No.) }\end{array}$ & $\begin{array}{l}\text { TN } \\
\text { (No.) }\end{array}$ & $\begin{array}{l}\text { FP } \\
\text { (No.) }\end{array}$ & $\begin{array}{l}\mathrm{FN} \\
\text { (No.) }\end{array}$ & $\begin{array}{c}\text { Sensitivity (\%) } \\
\text { (No.) }\end{array}$ & $\begin{array}{l}\text { Specificity (\%) } \\
\text { (No.) }\end{array}$ & $\begin{array}{l}\text { PPV (\%) } \\
\text { (No.) }\end{array}$ & $\begin{array}{l}\text { NPV (\%) } \\
\text { (No.) }\end{array}$ & $\begin{array}{c}\text { Accuracy (\%) } \\
\text { (No.) }\end{array}$ \\
\hline $\begin{array}{l}>5.0 \mathrm{~mm} \\
95 \% \mathrm{Cl}\end{array}$ & 23 & 45 & 0 & 0 & $\begin{array}{c}100(23 / 23) \\
86-100\end{array}$ & $\begin{array}{c}100(45 / 45) \\
92-100\end{array}$ & $\begin{array}{c}100(23 / 23) \\
86-100\end{array}$ & $\begin{array}{c}100(45 / 45) \\
92-100\end{array}$ & $\begin{array}{c}100(68 / 68) \\
95-100\end{array}$ \\
\hline $\begin{array}{c}3.1-5.0 \mathrm{~mm} \\
95 \% \mathrm{Cl}\end{array}$ & 35 & 45 & 0 & 0 & $\begin{array}{c}100(35 / 35) \\
90-100\end{array}$ & $\begin{array}{c}100(45 / 44) \\
92-100\end{array}$ & $\begin{array}{c}100(35 / 35) \\
90-100\end{array}$ & $\begin{array}{c}100(45 / 45) \\
92-100\end{array}$ & $\begin{array}{c}100(80 / 80) \\
94-100\end{array}$ \\
\hline $\begin{array}{l}\leq 3.0 \mathrm{~mm} \\
95 \% \mathrm{Cl}\end{array}$ & 16 & 45 & 1 & 1 & $\begin{array}{c}94(16 / 17) \\
73-99\end{array}$ & $\begin{array}{c}98(45 / 46) \\
89-100\end{array}$ & $\begin{array}{c}94(16 / 17) \\
73-99\end{array}$ & $\begin{array}{c}98(45 / 46) \\
89-100\end{array}$ & $\begin{array}{c}97(61 / 63) \\
89-100\end{array}$ \\
\hline
\end{tabular}

Note:-TP indicates true-positive; TN, true-negative; FP, false-positive; FN, false-negative; PPV, positive predictive value; NPV, negative predictive value. 

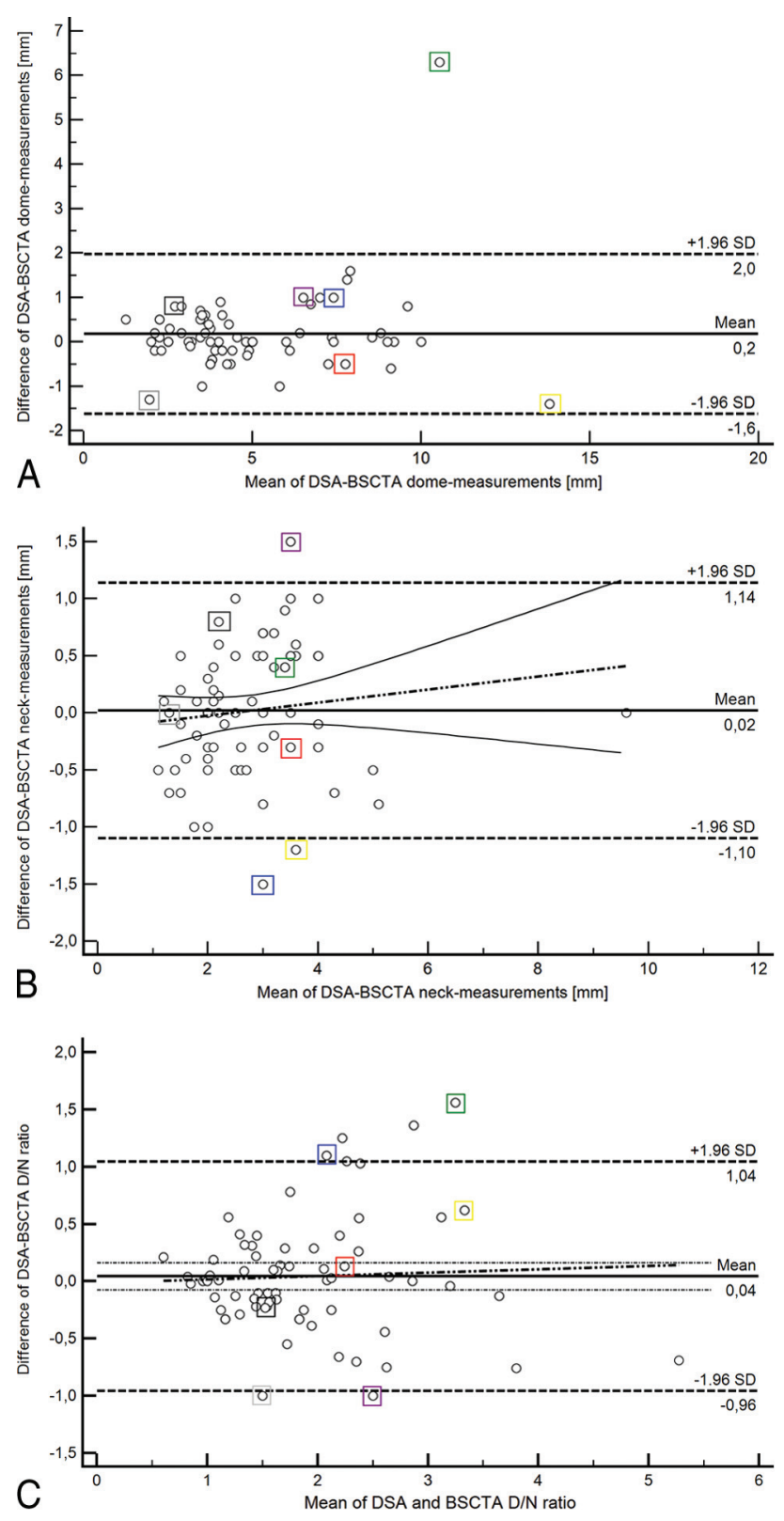

FIG 4. Bland-Altman plots show the relationship between differences and means of DSA and BSCTA in aneurysm dome $(A)$ and neck $(B)$ measurements and dome/neck ratios $(C)$. The black dotted line indicates the regression line of the differences. The 2 thin black lines represent the $95 \%$ confidence interval for the regression line of the differences. A, Bone subtraction CTA tends to overestimate aneurysm domes by $0.17 \mathrm{~mm}(95 \% \mathrm{Cl}, 0.04-0.39 \mathrm{~mm})$ and has a mild trend toward higher values for dome diameters with larger values. The colored rectangular boxes highlight manual measurements with interpolation of DSA results because DSA millimeter calibrations were not transferred with the other DSA data. The outlier case is 1 large 14.0- $\mathrm{mm}$ aneurysm that was overestimated by $6.0 \mathrm{~mm}$ and belongs to the manually calculated measurements. $B$, Bone-subtraction CTA measurements of the aneurysm neck are in good agreement with DSA $(0 \pm 1.96 \mathrm{~mm})$. Outlier cases are small $2.5-$ and $2.7-\mathrm{mm}$ aneurysms that were underestimated by -1.5 and $-1.2 \mathrm{~mm}$. The third outlier was a 2.7- $\mathrm{mm}$ aneurysm that was overestimated by $1.5 \mathrm{~mm}$. C, Bone-subtraction CTA slightly overestimates dome/neck ratios compared with DSA (mean, $0.04 ; 95 \% \mathrm{Cl}, 0.08-0.16$ ). Four of the 6 outliers belong to the manually calculated DSA measurements (colored rectangular boxes).
Table 5: Patient motion artifacts and performance on aneurysm detection

\begin{tabular}{|c|c|c|c|c|c|c|}
\hline \multirow[b]{2}{*}{ Method } & \multirow{2}{*}{$\begin{array}{c}\text { Intubated } \\
\text { Patients }\end{array}$} & \multicolumn{3}{|c|}{ Motion Artifacts } & \multicolumn{2}{|c|}{$\begin{array}{l}\text { Aneurysm } \\
\text { Detection }\end{array}$} \\
\hline & & Mild & Moderate & Severe & $\mathrm{FN}$ & FP \\
\hline DSA & 1 & 15 & 6 & 7 & 0 & 0 \\
\hline $\mathrm{CT}$ & 0 & 1 & 1 & 0 & 0 & 0 \\
\hline
\end{tabular}

Note:-F.FP indicates false-positive; FN, false-negative.

\section{DISCUSSION}

Our study confirmed a high accuracy of 16-detector row BSCTA in depicting and characterizing intracranial aneurysms. Immediate selection and planning of treatment was possible, even for complex and small aneurysms. This effect was true even for the relatively small amount of aneurysms that were close to bony structures. Furthermore, we demonstrated that the BSCTA method is already delivering high accuracy and robustness, even with widely available, outdated, 16-detector row multidetector CT hardware.

Therefore given the radiation-exposure reduction, BSCTA could replace diagnostic DSA. More advanced CT technology will probably perform as well or even better.

CTA has a high sensitivity and specificity in detecting intracranial aneurysms, ${ }^{8,9,11,12,17,19,20}$ but skull base structures can hide adjacent aneurysms. ${ }^{17,21}$ Bone-subtraction $\mathrm{CTA}^{22,23}$ has been developed to overcome this problem. A feasibility study ${ }^{22}$ and a study with 100 patients ${ }^{24}$ showed that BSCTA can improve the detection of vascular pathology closely adjacent to bony structures. These studies, however, did not use DSA as the reference standard to determine the diagnostic accuracy of BSCTA under clinical conditions. A recent 320-detector row BSCTA study evaluated the diagnostic accuracy of nonsubtracted and subtracted volumetric CTA data. ${ }^{12}$ The sensitivity for nonsubtracted CTA was $96.7 \%$ compared with $99.2 \%$ for subtracted CTA with $100 \%$ specificity for both.

Our findings are concordant with those in previous reports ${ }^{8-11}$ and compare well with a meta-analysis of twelve 16-detector row BSCTA studies. ${ }^{10}$ The sensitivity and specificity of BSCTA for small aneurysms $(<3.0 \mathrm{~mm})$ were lower than those in our population.

Our study results also compare well with 8 pooled $64-$ detector row BSCTA studies. ${ }^{10}$ Similar results were seen in a $64-$ detector row multidetector CT study with 89 patients. ${ }^{7}$ Our study results are also in line with those in 2 other studies by using modern dual-source and $320-$ detector row BSCTA. ${ }^{12}$

In our study, 1 aneurysm of $<3 \mathrm{~mm}(2.5 \mathrm{~mm})$ in diameter was missed by DSA (Fig 3). Another $<3-\mathrm{mm}(1.7 \mathrm{~mm}$ ) aneurysm was initially missed by BSCTA (Fig 2). In both cases, these findings did not change the treatment strategy. Both methods have a small risk of missing small aneurysms. The diagnostic accuracy of DSA was limited by complex vascular anatomy (vessel trifurcation, small diameter of $<3 \mathrm{~mm}$ ) and inadequate projections due to patient motion. In a study with 50 patients presenting with diffuse aneurysmal pattern, 2 aneurysms were missed initially by DSA and BSCTA. ${ }^{25}$

False-positive cases on both 16- and 64-detector row BSCTA can be explained a by focal venous plexus overlying the MCA. ${ }^{17}$ Venous contrast has been described as a potential source of error. ${ }^{26,27}$ Venous enhancement is, however, not a crucial factor in the detection of cerebral aneurysms, except for extensive en- 
hancement of the cavernous sinus. ${ }^{28}$ In contrast, with 16 -detector row CTA and bolus triggering, arterial and venous structures can be distinguished by their different attenuations. Whether modern, wide-detector ( $>64-$ detector row), multidetector CT is capable of further improving the diagnostic accuracy of BSCTA, beyond the known 16- and 64-detector row multidetector CT results, needs to be investigated. With wider $\mathrm{z}$-coverage, the negative effect of scatter radiation and conebeam artifacts increases and may not lead to a further gain in accuracy. ${ }^{29,30}$

The most common and well-studied geometric determinant of treatment decisions and outcome is the dome-to-neck ratio. ${ }^{21}$ Aspects of aneurysm geometry such as shape, size, dome-to-neck ratio, and location and the relationship to the parent vessels all may impact treatment decisions. ${ }^{31,32}$

Interobserver agreement in our study was somewhat higher than that reported previously. ${ }^{11,17,19,26}$ Intraobserver agreement was excellent as in another study by Lu et al. ${ }^{11}$

We had excellent interreader agreement for aneurysm identification and configuration. Our results are in line with those in other studies, ${ }^{17,26}$ even with a recent study with 320 -detector row $\mathrm{BSCTA}^{33}$ comparing CTA with intraoperative observations.

Only 1 study evaluated the $\mathrm{D} / \mathrm{N}$ ratios in a comparable fashion, but not in the same level of detail. ${ }^{26}$ In contrast to our results, this study reported a general overestimation of $\mathrm{D} / \mathrm{N}$ ratio with 16-detector row BSCTA due to partial volume sampling effects. ${ }^{24}$ Considerable overestimation of the $\mathrm{D} / \mathrm{N}$ ratio of aneurysms may have led to therapeutic option changes. ${ }^{26}$ Endovascular treatment of wide-neck or "difficult" aneurysms requires special techniques such as balloon- or stent-assisted coiling. ${ }^{21}$ Because we did not document the decisions of our interventionists and neurosurgeons, we do not know to what extent the information provided by BSCTA has influenced patient management. We can state, however, that BSCTA assessed the site and shape of cerebral aneurysms as accurately as DSA.

Radiation is an important factor for patients with $\mathrm{SAH},{ }^{34}$ because many are younger and need repeat brain imaging. Our BSCTA dose values were below the European reference value (2.4 $\mathrm{mSv}$ ) for CT angiography of the brain. ${ }^{35}$ Our average DSA radiation dose remained at the lower limit of doses reported for DSA (3.5-6.5 mSv) ${ }^{36}$ A disadvantage of BSCTA is the radiation dose, due to 2 consecutive scans, which means an increase of exposure by $20 \%-25 \%$ above the level of standard CTA according to our and others' experience. ${ }^{37,38}$ Low-dose settings for nonenhanced CT are acceptable in achieving an effective dose below $3 \mathrm{mSv}$. Recently new approaches are being evaluated to replace the lowdose NECT with either a standard-dose NECT or a late venous CT dataset, to receive the subtraction bone mask. ${ }^{39-41}$ Optimally, the work-up of SAH before the selection of a method of aneurysm treatment should be exclusively noninvasive diagnostics. In contrast to diagnostic DSA, BSCTA can reduce the door-to-treatment time by providing the relevant information instantly. ${ }^{17,26}$ It may even prevent DSA in cases with a clear indication for clipping. ${ }^{26,27,42}$ The accuracy of BSCTA being comparable with DSA now allows clipping of these aneurysms without additional DSA when transarterial intervention is not possible. In cases where the first CTA does not show the cause of SAH, it is not necessary to perform DSA. A second CTA is sufficient.

Moreover, BSCTA can exclude an aneurysm as the cause of spontaneous SAH. ${ }^{17}$

Our study has limitations. Although BSCTA was feasible in almost all patients, we could recruit patients only when the involved neuroradiologist was on duty and had ordered the new BSCTA protocol. We cannot safely exclude the possibility that the patients identified by study neuroradiologists were different from those with SAH seen by a radiologist not familiar with the study. We aimed to consecutively identify all patients with acute SAH and without previous aneurysm treatment, which means we had no knowledge of the source of bleeding. We thought this population was the best to study the accuracy of BSCTA. We lost patients for the study when radiologists on call were not yet familiar with the study protocol. We think, however, that the patients we recruited represented the population typical for acute SAH. Consequently, we extended the observation period to reach the planned sample size.

In our institution, we now recommend BSCTA in addition to NECT as the first imaging technique in patients with acute SAH. Our neurosurgeons clip aneurysms solely on the basis of this information in urgent cases that cannot be coiled. We have reduced the DSA protocol before intervention to the artery affected and do not further perform panangiographies. We further recommend repeat BSCTA in cases of CTA with negative findings in patients with SAH, but we admit that not all of our neurosurgeons consistently follow this advice.

\section{CONCLUSIONS}

The widely available 16-detector row BSCTA allows reliable and accurate detection and characterization of cerebral aneurysms in patients with acute $\mathrm{SAH}$ and thus can guide treatment decisions faster and more efficiently. If the location and shape of aneurysms favor surgical clipping, an additional DSA is no longer necessary because all information needed by neurosurgeons is provided. If coiling is preferred, complete diagnostic 4-vessel panangiography is no longer routinely required. Interventionists can focus DSA on the site of the symptomatic aneurysm. This new strategy will not only reduce the risks and radiation dose for patients and physicians, but also reduce cost and time. More modern CT technology may make BSCTA more efficient, more standardized, and finally easier to apply.

Disclosures: Peter Aulbach—RELATED: Support for Travel to Meetings for the Study or Other Purposes: Siemens, Comments: Siemens, as my employer, supports my studies through payments for traveling to the university and back. It is just the cost for hotel, car, and fuel; UNRELATED: Employment: Siemens, Comments: I am an employee of Siemens. My PhD is tolerated there as part of my personal development. My employer just compensates the cost for me to travel to the University (car, fuel, and hotel). Additionally, Siemens allows me to spend time at the university if it is not too many days. As long as my work output does not suffer, they tolerate it. Rüdiger von Kummer-UNRELATED: Board Membership: Lundbeck; Consultancy: Lundbeck, Penumbra, Covidien, Boehringer Ingelheim; Payment for Lectures (including service on Speakers Bureaus): Penumbra; Royalties: Elsevier, Springer, Comments: book chapter authorship.

\section{REFERENCES}

1. Connolly ES Jr, Rabinstein AA, Carhuapoma JR, et al; American Heart Association Stroke Council, Council on Cardiovascular Radi- 
ology and Intervention, Council on Cardiovascular Nursing, Council on Cardiovascular Surgery and Anesthesia, Council on Clinical Cardiology. Guidelines for the management of aneurysmal subarachnoid hemorrhage: a guideline for healthcare professionals from the American Heart Association/American Stroke Association. Stroke 2012;43:1711-37 CrossRef Medline

2. Dawkins AA, Evans AL, Wattam J, et al. Complications of cerebral angiography: a prospective analysis of 2,924 consecutive procedures. Neuroradiology 2007;49:753-59 CrossRef Medline

3. Fifi JT, Meyers PM, Lavine SD, et al. Complications of modern diagnostic cerebral angiography in an academic medical center. $J$ Vasc Interv Radiol 2009;20:442-47 CrossRef Medline

4. Velthuis BK, Van Leeuwen MS, Witkamp TD, et al. Computerized tomography angiography in patients with subarachnoid hemorrhage: from aneurysm detection to treatment without conventional angiography. J Neurosurg 1999;91:761-67 CrossRef Medline

5. Rinkel GJ, van Gijn J, Wijdicks EF. Subarachnoid hemorrhage without detectable aneurysm: a review of the causes. Stroke 1993;24: 1403-09 CrossRef Medline

6. Saitoh H, Hayakawa K, Nishimura K, et al. Rerupture of cerebral aneurysms during angiography. AJNR Am J Neuroradiol 1995;16: 539-42 Medline

7. Li Q, Lv F, Li Y, et al. Evaluation of 64-section CT angiography for detection and treatment planning of intracranial aneurysms by using DSA and surgical findings. Radiology 2009;252:808-15 CrossRef Medline

8. Zhang LJ, Wu SY, Niu JB, et al. Dual-energy CT angiography in the evaluation of intracranial aneurysms: image quality, radiation dose, and comparison with 3D rotational digital subtraction angiography. AJR Am J Roentgenol 2010;194:23-30 CrossRef Medline

9. Zhang LJ, Wu SY, Poon CS, et al. Automatic bone removal dualenergy CT angiography for the evaluation of intracranial aneurysms. J Comput Assist Tomogr 2010;34:816-24 CrossRef Medline

10. Menke J, Larsen J, Kallenberg K. Diagnosing cerebral aneurysms by computed tomographic angiography: meta-analysis. Ann Neurol 2011;69:646-54 CrossRef Medline

11. Lu L, Zhang LJ, Poon CS, et al. Digital subtraction CT angiography for detection of intracranial aneurysms: comparison with threedimensional digital subtraction angiography. Radiology 2012;262: 605-12 CrossRef Medline

12. Chen W, Xing W, Peng Y, et al. Cerebral aneurysms: accuracy of 320detector row nonsubtracted and subtracted volumetric $\mathrm{CT}$ angiography for diagnosis. Radiology 2013;269:841-49 CrossRef Medline

13. Papke K, Kuhl CK, Fruth M, et al. Intracranial aneurysms: role of multidetector CT angiography in diagnosis and endovascular therapy planning. Radiology 2007;244:532-40 CrossRef Medline

14. Westerlaan HE, van Dijk JMC, van Dijk MJ, et al. Intracranial aneurysms in patients with subarachnoid hemorrhage: $\mathrm{CT}$ angiography as a primary examination tool for diagnosis-systematic review and meta-analysis. Radiology 2011;258:134-45 CrossRef Medline

15. Agid R, Willinsky RA, Farb RI, et al. Life at the end of the tunnel: why emergent CT angiography should be done for patients with acute subarachnoid hemorrhage. AJNR Am J Neuroradiol 2008;29:e45; author reply e46-47 CrossRef Medline

16. Fox AJ, Symons SP, Aviv RI. CT angiography is state-of-the-art first vascular imaging for subarachnoid hemorrhage. AJNR Am J Neuroradiol 2008;29:e41-42; author reply e46-47 CrossRef Medline

17. McKinney AM, Palmer CS, Truwit CL, et al. Detection of aneurysms by 64-section multidetector CT angiography in patients acutely suspected of having an intracranial aneurysm and comparison with digital subtraction and 3D rotational angiography. AJNR Am J Neuroradiol 2008;29:594-602 CrossRef Medline

18. Donmez H, Serifov E, Kahriman G, et al. Comparison of 16-row multislice CT angiography with conventional angiography for detection and evaluation of intracranial aneurysms. Eur J Radiol 2011; 80:455-61 CrossRef Medline

19. Romijn M, Gratama van Andel HA, van Walderveen MA, et al. Diagnostic accuracy of CT angiography with matched mask bone elim- ination for detection of intracranial aneurysms: comparison with digital subtraction angiography and 3D rotational angiography. AJNR Am J Neuroradiol 2008;29:134-39 CrossRef Medline

20. Li Q, Lv F, Li Y, et al. Subtraction CT angiography for evaluation of intracranial aneurysms: comparison with conventional CT angiography. Eur Radiol 2009;19:2261-67 CrossRef Medline

21. Brinjikji W, Cloft HJ, Kallmes DF. Difficult aneurysms for endovascular treatment: overwide or undertall? AJNR Am J Neuroradiol 2009;30:1513-17 CrossRef Medline

22. Lell MM, Ruehm SG, Kramer M, et al. Cranial computed tomography angiography with automated bone subtraction: a feasibility study. Invest Radiol 2009;44:38 - 43 CrossRef Medline

23. Lell M, Anders K, Klotz E, et al. Clinical evaluation of bone-subtraction CT angiography (BSCTA) in head and neck imaging. Eur Radiol 2006;16:889-97 CrossRef Medline

24. Morhard D, Fink C, Becker C, et al. Value of automatic bone subtraction in cranial CT angiography: comparison of bone-subtracted vs. standard CT angiography in $\mathbf{1 0 0}$ patients. Eur Radiol 2008;18:974-82 CrossRef Medline

25. Agid R, Andersson T, Almqvist H, et al. Negative CT angiography findings in patients with spontaneous subarachnoid hemorrhage: when is digital subtraction angiography still needed? AJNR Am J Neuroradiol 2010;31:696-705 CrossRef Medline

26. Yoon DY, Lim KJ, Choi CS, et al. Detection and characterization of intracranial aneurysms with 16-channel multidetector row CT angiography: a prospective comparison of volume-rendered images and digital subtraction angiography. AJNR Am J Neuroradiol 2007;28:60-67 Medline

27. Agid R, Lee SK, Willinsky RA, et al. Acute subarachnoid hemorrhage: using 64-slice multidetector CT angiography to "triage" patients' treatment. Neuroradiology 2006;48:787-94 CrossRef Medline

28. Lell MM, Anders $\mathrm{K}$, Uder M, et al. New techniques in CT angiography. Radiographics 2006;26(suppl 1):S45-62 CrossRef Medline

29. Li B, Toth TL, Hsieh J, et al. Simulation and analysis of image quality impacts from single source, ultra-wide coverage CT scanner. J Xray Sci Technol 2012;20:395-404 CrossRef Medline

30. Boas FE, Fleischmann D. CT artifacts: causes and reduction techniques. Imaging Med 2012;4:229-40 CrossRef

31. Gonzalez N, Sedrak M, Martin N, et al. Impact of anatomic features in the endovascular embolization of 181 anterior communicating artery aneurysms. Stroke 2008;39:2776-82 CrossRef Medline

32. Brinjikji W, Cloft H, Lanzino G, et al. Comparison of 2D digital subtraction angiography and $3 \mathrm{D}$ rotational angiography in the evaluation of dome-to-neck ratio. AJNR Am J Neuroradiol 2009;30: 831-34 CrossRef Medline

33. Hayashida E, Sasao A, Hirai T, et al. Can sufficient preoperative information of intracranial aneurysms be obtained by using $\mathbf{3 2 0}$ row detector CT angiography alone? Jpn J Radiol 2013;31:600-07 CrossRef Medline

34. Gelfand AA, Josephson SA. Substantial radiation exposure for patients with subarachnoid hemorrhage. J Stroke Cerebrovasc Dis 2011; 20:131-33 CrossRef Medline

35. European Commission. European guidelines on quality criteria for computed tomography. Report EUR 16262. Brussels, Belgium: European Commission, 1999

36. Westerlaan HE, Gravendeel J, Fiore D, et al. Multislice CT angiography in the selection of patients with ruptured intracranial aneurysms suitable for clipping or coiling. Neuroradiology 2007;49:9971007 CrossRef Medline

37. Venema HW, Hulsmans FJ, den Heeten GJ. CT angiography of the circle of Willis and intracranial internal carotid arteries: maximum intensity projection with matched mask bone elimination-feasibility study. Radiology 2001;218:893-98 CrossRef Medline

38. Tomandl BF, Hammen T, Klotz E, et al. Bone-subtraction CT angiography for the evaluation of intracranial aneurysms. AJNR Am J Neuroradiol 2006;27:55-59 Medline

39. Huang A, Lee CW, Yang CY, et al. Using standard nonenhanced 
axial scans for cerebral CT angiography bone elimination: feasibility study. Invest Radiol 2010;45:225-32 CrossRef Medline

40. Lell MM, Ditt H, Panknin C, et al. Cervical CT angiography comparing routine noncontrast and a late venous scan as masks for automated bone subtraction: feasibility study and examination of the influence of patient motion on image quality. Invest Radiol 2008;43: 27-32 CrossRef Medline
41. Gratama van Andel HA, Venema HW, Streekstra GJ, et al. Removal of bone in CT angiography by multiscale matched mask bone elimination. Med Phys 2007;34:3711-23 CrossRef Medline

42. Pozzi-Mucelli F, Bruni S, Doddi M, et al. Detection of intracranial aneurysms with 64 channel multidetector row computed tomography: comparison with digital subtraction angiography. Eur J Radiol 2007;64:15-26 CrossRef Medline 